Review Article

\title{
Intestinal Stem Cell Niche Insights Gathered from Both In Vivo and Novel In Vitro Models
}

\author{
Nikolce Gjorevski ${ }^{1}$ and Paloma Ordóñez-Morán ${ }^{2}$ \\ ${ }^{1}$ Laboratory of Stem Cell Bioengineering, Institute of Bioengineering, School of Life Sciences (SV) and School of Engineering (ST), \\ École Polytechnique Fédérale de Lausanne (EPFL), Lausanne, Switzerland \\ ${ }^{2}$ Swiss Institute for Experimental Cancer Research (ISREC), École Polytechnique Fédérale de Lausanne (EPFL), Lausanne, Switzerland
}

Correspondence should be addressed to Paloma Ordóñez-Morán; paloma.ordonez@epfl.ch

Received 31 March 2017; Accepted 3 July 2017; Published 7 September 2017

Academic Editor: Karen Liu

Copyright (C) 2017 Nikolce Gjorevski and Paloma Ordóñez-Morán. This is an open access article distributed under the Creative Commons Attribution License, which permits unrestricted use, distribution, and reproduction in any medium, provided the original work is properly cited.

\begin{abstract}
Intestinal stem cells are located at the base of the crypts and are surrounded by a complex structure called niche. This environment is composed mainly of epithelial cells and stroma which provides signals that govern cell maintenance, proliferation, and differentiation. Understanding how the niche regulates stem cell fate by controlling developmental signaling pathways will help us to define how stem cells choose between self-renewal and differentiation and how they maintain their undifferentiated state. Tractable in vitro assay systems, which reflect the complexity of the in vivo situation but provide higher level of control, would likely be crucial in identifying new players and mechanisms controlling stem cell function. Knowledge of the intestinal stem cell niche gathered from both in vivo and novel in vitro models may help us improve therapies for tumorigenesis and intestinal damage and make autologous intestinal transplants a feasible clinical practice.
\end{abstract}

\section{Introduction}

The intestine represents the most vigorously renewing adult tissue, which undergoes rapid turnover in order to prevent damage from stress factors; its tissue-specific stem cells are essential for tissue homeostasis in the adult organism [1]. These undifferentiated cells residing at the bottom of the crypts of Lieberkühn are able to produce a large number of differentiated progeny as well as to selfrenewal. Due to their relevant function, many efforts have been done in the last years to define the exact localization of the intestinal stem cells and its properties. There is now evidence that at least two types of stem cells coexist in the small intestine. Best characterized are the leucine-richrepeat-containing G-protein-coupled receptor 5-expressing $\left(\operatorname{Lgr}^{+}{ }^{+}\right)$stem cells which divide approximately every 24 hours, and they are interspersed between the terminally differentiated Paneth cells [2]. The Lgr5 gene was selected from a panel of intestinal Wnt targets for its restricted crypt expression (columnar base cells, CBC) and was identified as a marker gene of stem cells in the small intestine and colon [2]. Very recent findings have found that $\mathrm{Lgr}^{+}$stem cell population is not homogenous. The expression of the RNA-binding protein Mex3a labels a slowly cycling subpopulation of $\mathrm{Lgr}^{+}$ISCs that contribute to all intestinal lineages. Thus, Mex3a defines a reservelike ISC population within the $\mathrm{Lgr}^{+}$compartment [3]. The second type of stem cells are located at the +4 position of the intestinal crypt and are called label-retaining cells (LRCs) as they show long-term label retention upon irradiation damage and pulse labeling with BrdU. These cells remain quiescent and act as a reserve population that can give rise to all intestinal cell lineages after tissue damage [4-8]. Some reports point out that there is an apparent dichotomy between quiescent versus cycling stem cells that in fact reflect a continuum of phenotypes dictated by different thresholds of expression of key regulators (e.g., signals and/or transcription factors) that modulate stem-like functions [7, 9-13]. Future experiments for a better identification of these mechanisms and the features of the +4 LRC stem 


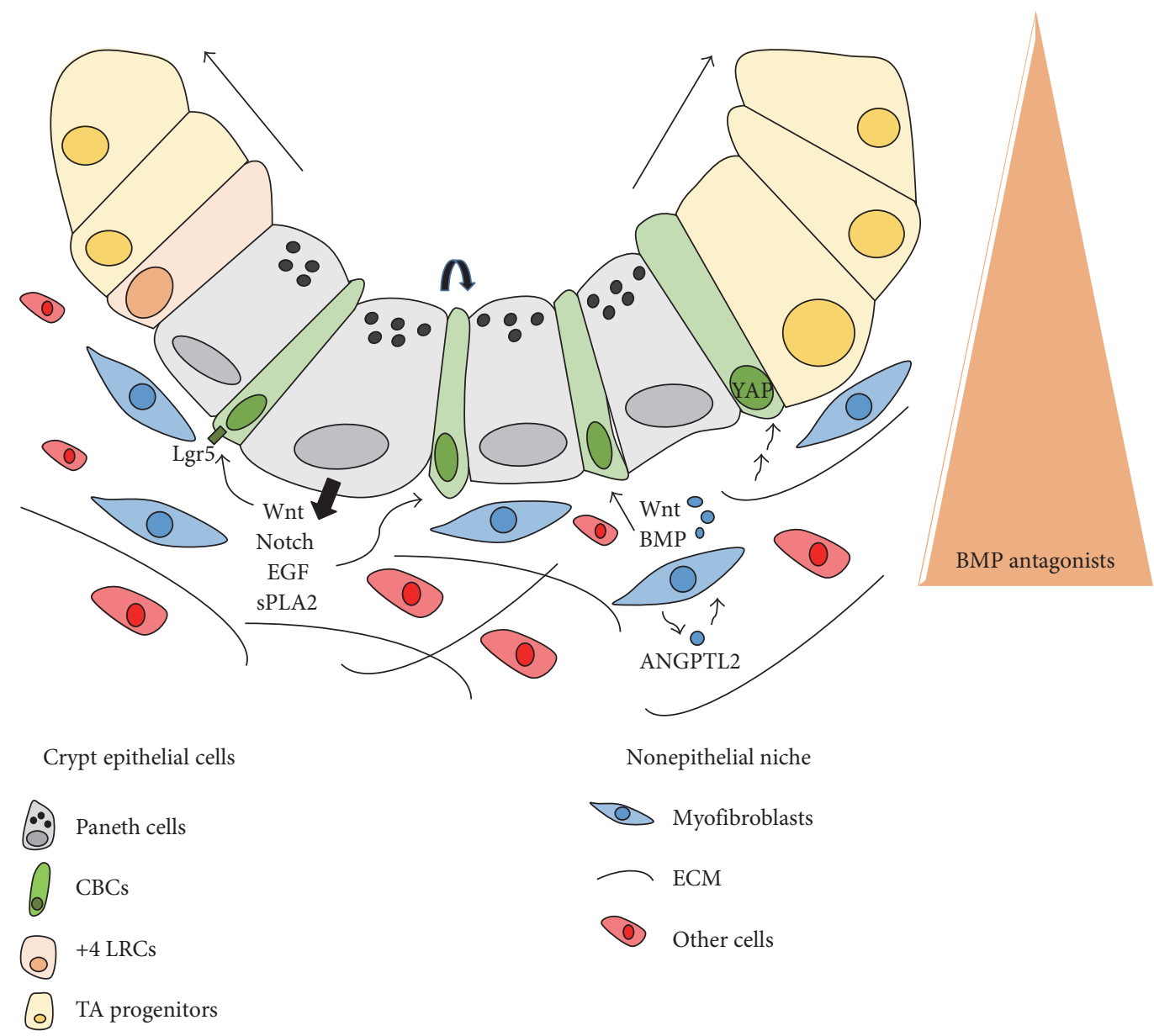

Figure 1: The stem cell niche of the small intestine. Epithelial and nonepithelial environments support the signals required for stem cell maintenance. Among them, Wnt and Notch signaling have been defined as major determinants for stem cell self-renewal, for proliferation/differentiation of stem cells in the crypt. Stromal BMP antagonists regulate the crypt-villus axis, and the extracellular cell matrix (ECM) support signals that control stem cell fate. Other cells: neural, immune, and endothelial cells. TA: transit-amplifying progenitors; sPLA2: secreted phospholipases A2.

cell populations are still needed in order to understand the capacity of the intestinal tissue to induce a regenerative response under (radiation induced) tissue injury. In this review, we will mostly focus on the in vivo and in vitro models for intestinal CBC stem cell niche.

Control of proliferation, self-renewal, and lineage specification of the stem cells in the crypt are believed to be directed by an actively regulated process based on cell-cell and cellstroma interactions [14]. The ISC niche or microenvironment is composed of epithelial and underlying nonepithelial cells within the lamina propia populated by stromal, immune, endothelial, and neural cells that support paracrine and/or autocrine signaling (Figure 1). The ISC niche also comprises the extracellular matrix (ECM), a highly dynamic structure that continuously undergoes controlled remodelling, mediated by metalloproteinases that are responsible for ECM degradation [15]. The ECM interacts with the different cells in the niche to regulate stem cell fate [16] (Figure 1). Overall, the components of the niche tightly modulate Wnt, Notch, epidermal growth factor (EGF), bone morphogenic protein (BMP)/transforming growth factor
(TGF) $\beta$, and Hedgehog signaling pathways to maintain proliferation/differentiation balance [17-19].

Functional analysis of stem cells and their environment has been hampered by a lack of suitable in vitro systems allowing long-term culture and until some years ago, the only possible strategy to analyse such interactions for a potential role in intestinal development, homeostasis, damage or tumorigenesis was the time-consuming tissue-specific mouse models. For example, Achaete-scute complex homolog 2 (Ascl2) was reported to be responsible for controlling intestinal stem cell fate by using transgenic mice [20]. In 2009, two groups developed a three-dimensional (3D) culture model of freshly isolated crypt cells from murine small intestine and colon [21-23], and later this method was set up for human samples [24, 25]. These assays maintain basic crypt-villus physiology and permit long-term intestinal epithelial expansion as sphere-like organoids. The stem cells are embedded in Matrigel, a gelatinous protein mixture secreted by mouse sarcoma cells containing structural proteins such as laminin, entactin, and collagen in combination with several growth stimuli essential for crypt proliferation (the Wnt agonist R- 
spondin1, EGF, and the BMP inhibitor Noggin). Singlesorted Lgr $5^{+}$stem cells are sufficient to give rise to organoids in culture which contain all differentiated lineages: Paneth cells at the base of the crypt and enteroendocrine, goblet cells, and enterocytes that migrate upwards the villus. Importantly, these cultures allow ex vivo monitoring intestinal stem cell function with respect to self-renewal and production of rapidly dividing crypt progenitor cells and differentiated lineages and are therefore comparable to the in vivo situation [21]. In this review, we will compare in vivo models to the most novel in vitro technology which will improve in the next years our understanding of stem cell behavior.

\section{In Vivo Models of the Stem Cell Niche}

2.1. CBC Epithelial Niche. Stem cells require the support of neighbouring epithelial cells to maintain their function. The epithelial niche modulates several signaling cascades, the Wnt pathway being one of the main regulators of stem cell self-renewal. Genetic deletion of the Wnt pathway's main players ( $\beta$-catenin, Tcf4 knockout models) or ectopic expression of the secreted Wnt antagonist Dickkopf-1 (Dkk1) disrupts intestinal epithelial homeostasis, leading to crypt loss, reduced proliferation, and altered differentiation [26-28]. Similarly, overactivation of the Wnt pathway in mice, by overexpression of the Wnt agonists roof plate-specific spondin 1 (R-spondin 1 ) and R-spondin 3 or by deleting Adenomatous polyposis coli $(A p c)$, drives hyperplasia and increases the expansion of intestinal stem cell niche [29-31]. The Wnt target genes, EphB2, EphB3, and their ligands ephrins, are key coordinators of migration and proliferation in the stem cell niche. EphB knockouts show that these proteins determine cell positioning along the crypt-villus axis in the intestinal epithelium [32]. Furthermore, EphB signaling promotes cell-cycle reentry of progenitor cells and contributes to the mitogenic activity in the adult mouse small intestine and colon [33].

"What is the main source of epithelial Wnt signals within the intestine?" The secretory Paneth cells adjacent to CBCs secrete Wnt3, and they constitute an important part of the small intestinal stem cell niche. They are known to produce bactericidal products such as lysozyme and cryptdins/defensins, and in addition, they can also produce TGF $\alpha$, Notch (Dll4), and EGF factors that regulate stem cell maintenance $[34,35]$. Reduction of number of Paneth cells in Gfi1 ${ }^{-/-}$ mouse model, transgenic expression of diphtheria toxin A under the Paneth cell-specific cryptdin 2 promoter (CR2tox176), and conditional deletion of Sox9 showed that the stem cells were coincidently decreased in number [34]. Some studies indicate that these cells are dispensable for small intestinal homeostasis; however, it should be determined in additional mouse models able to also achieve a total disruption of Paneth cells [36]. Although, this exact type of cells are not present in the colon, Clarke's group found goblet cells interdigitated with $\operatorname{Lgr} 5^{+}$stem cells that contained a distinct cKit/CD $117^{+}$crypt base subpopulation which expressed the Notch ligands Delta-like (Dll) 1, Dll4, and EGF. In vivo, this colonic cKit population was regulated by Notch signaling [37]. Later on, Clevers lab described equivalent cells called regenerating islet-derived family member (Reg) $4^{+}$-expressing deep crypt secretory (DCS) cells (called Paneth/gobletlike cells) that are intermingled with the $\operatorname{Lgr} 5^{+}$colonic stem cells at the base of the crypt. These cells also produce Wnt and Notch factors to support essential growth and maintenance signals. In these mouse experiments, the ablation of these types of cells resulted in loss of stem cell function and disruption of colon homeostasis [38]. When Paneth or DCS cells were sorted together with $\operatorname{Lgr} 5^{+}$cells, the signals provided by them markedly increased differentiation and organoid growth from a single stem cell in the in vitro culture $[34,38]$. Novel data have revealed that Paneth and DCS cells are also secreting the phospholipases A2 (sPLA2s) which inhibit Wnt pathway through intracellular activation of Yap1. Importantly, this cascade affects stem cell niche during homeostasis [39].

Several Notch mouse models evidence the impact of this pathway in epithelial stem cell niche. Indeed, $\operatorname{Lgr} 5^{+}$stem cells are critically dependent on Notch, which depend on direct cell-cell contact as the Paneth cells are the main sources of Notch signals [40-46]. This is the case of simultaneous inactivation of Dll1 and Dll4 which resulted in the complete conversion of proliferating progenitors into postmitotic goblet cells, concomitant with the loss of $\operatorname{Lgr} 5^{+}$SCs [41]. A negative regulatory mechanism of Wnt and Notch influencing intestinal stem cells in the gut was nicely shown by Tian et al. When Notch pathway was blocked, it perturbed intestinal stem cell function by causing a derepression of the Wnt pathway, leading to misexpression of prosecretory genes. Then, attenuation of Wnt rescued the phenotype associated with Notch blockade [43].

Other studies show Leucine-rich repeats and immunoglobulin-like domains (Lrig) 1, a direct Myc target gene as part of a negative feedback loop that modulates the proliferation of intestinal progenitor cells. Lrig knockout mouse induces upregulation of EGFR, ErbB2, and ErbB3 promoting downstream activation of c-Myc within intestinal stem and progenitor cells $[17,47]$. The EGF pathway affects stem cell function by regulating the phosphoinositide 3kinase (PI3K), mitogen-activated protein kinase (MAPK), and/or protein kinase $\mathrm{C}$ (PKC) pathways among other cascades [48]. These and further studies on this direction may lead to next-generation stem cell-based therapies.

2.2. CBC Nonepithelial Niche. Vigorous crosstalk between the epithelium and the underlying nonepithelial niche is required to define the crypt-villus axis. It is well established that mesenchymal cells secrete BMP antagonists such as Gremlin 1 and Gremlin 2 at the bottom of the crypts which supports compartmentalization [49]. Thus, BMP signaling is inhibited for a right intestinal epithelial renewal. Indeed, mouse transgenic overexpression of the BMP antagonist Noggin affects crypt expansion and increased stem cell numbers [50, 51]. Moreover, genetic models carrying BMPR1A inactivation or deficiency of its downstream effector PTEN show an inhibition of BMP signaling that enhances AKT activation and an increase in Wnt signaling [1, 50]. Hedgehog signaling is also involved in this crosstalk by modulating stromal BMPs [52]. The gradient of Wnt and BMP pathway 
by diffusion of ligands along the crypt acts as a balance of cell differentiation/proliferation. Wnt is higher at the crypt base, whereas BMP pathway, which inhibits proliferation, has an opposite pattern of expression [1] (Figure 1).

Mesenchymal cells also secrete Wnt proteins, and $\mathrm{R}$-spondins have been detected in the intestinal stroma [19, 53-55]. Experiments by using inducible mouse deletion (only epithelial cells) of Porcupine (an endoplasmic reticulum resident $\mathrm{O}$-acyltransferase essential for the secretion and activity of all Wnts) showed that the cells had normal proliferation and differentiation, indicating that epithelial Wnt is dispensable for stem cell maintenance. Then, it was observed that intestinal stromal cells endogenously expressing Wnts and R-spondin3 support the growth of Porcupine-deficient organoids ex vivo, pointing out that stromal production of Wnts can fully support murine intestinal homeostasis [56].

All these data suggest that Wnt signals from Paneth cells can be replaced by stromal ones, so nonepithelial activation of Wnt pathway may support intestinal stem cell maintenance. On this direction, recent studies show that a subpopulation of mesenchymal cells marked by the winged-helix transcription factor Foxl1 is critical in maintaining stem cells. These cells produce Wnt factors, and their ablation reduces crypt growth. However, there is a need to better identify this subset of mesenchymal cells [57]. In addition, the autocrine secretion of ANGPTL2 by subepithelial myofibroblasts affects BMP production which then modulates intestinal organoid growth and size. Moreover, intestinal damage of Angptl2 knockout mice reduces CBCs and influences Wnt pathway; however, ANGPTL2 is dispensable for intestinal homeostasis [58] (Figure 1).

Remarkably, some other cell populations in the niche (immune, endothelial, and neural cells) also influence the stem cell behaviour by modulating the different signaling cascades. They secrete growth factors, cytokines, and ligands that alter the stem cell fate $[19,59]$. Upcoming work is required to reveal a more detailed comprehension of the interplay and components of this complex cellular network.

\section{In Vitro Models of the ISC Niche}

3.1. Intestinal Organoids. Animal models have provided invaluable insight into the nature and hallmarks of the intestinal stem cell, as well as the set of microenvironmental inputs that govern its behavior and constitute the intestinal stem cell (ISC) niche. However, in addition to ethical and practical considerations, animal-based studies suffer multiple limitations in the scope of scientific questions they can address. In particular, mouse models generally do not afford the dynamic and multifactorial observation and control that are required for securing comprehensive understanding of ISCs and their niche. Further, whereas the mouse intestine is an adequate approximation of its human counterpart, several crucial developmental and histological differences exist [60], and mouse-based studies may fall short in providing insight that is also relevant for humans. In vitro models of the ISC niche circumvent these problems by offering a level of accessibility and tractability that is difficult or impossible to achieve in vivo.

Driven by both basic research and therapeutic objectives, researchers have cultured stem cells in vitro for several decades. A decade ago, Sasai demonstrated that, aside from directing pluripotent stem cells to commit toward a certain lineage, thus obtaining populations of differentiated cells, stem cells and their progeny can follow their innate developmental programs and self-organize into structures that mimic multiple histological and functional aspects of real organs [61, 62]. These organ mimetics generated in vitro were termed organoids.

Intestinal organoids, or "miniguts", generated in the laboratory of Hans Clevers, were among the first types of stem cell-derived organoids reported $[21,24]$. Sato et al. showed that intestinal crypts or single-dissociated Lgr5-expressing ISCs embedded in Matrigel and provided with niche signals, including R-spondin1, Noggin, and EGF, not only survive and proliferate but also undergo morphogenesis and differentiation to produce structures that approximate the adult intestine: crypt-like projections radiate outward from a spherical epithelial structure that surrounds a central lumen. In addition to cycling ISCs, housed at the proximal ends of the crypt-like buds, intestinal organoids contain all differentiated intestinal cell types, which are represented at the ratios found in the native intestine [63] and in spatial arrangements that closely mimic the patterning of the crypt-villus axis. Importantly, these structures reconstitute the principal geometric, architectural, and cellular hallmarks of the ISC niche-Lgr5-expressing ISCs are attached to a basement membrane-like hydrogel. ISCs and Paneth cells are represented in numbers and ratios reflecting those in vivo, thus forming a bud structure of similar in shape and size to those of the intestinal crypt. Small modifications of the culture protocol-notably, the addition of Wnt3a-allow for the culture of adult human ISC-derived intestinal organoids [24]. It should be emphasized, however, that these structures feature a round, cystic architecture, thus missing the crypt-like domains of mouse intestinal organoids.

In addition to adult ISCs, intestinal organoids have been generated from induced pluripotent stem cells (PSCs), using a protocol inspired by human embryonic development [64]. PSCs were first treated with activin A to induce the formation of definitive endoderm, which was then steered toward mid/hindgut fates by treatment with FGF4 and Wnt3a. Culturing of the resulting mid/hindgut spheroids in Matrigel, under conditions used for the culture of adult ISCs and crypts [21], gave rise to intestinal organoids. Notably, PSC-derived human organoids are organized into cryptand villus-like domains, contain the major differentiated epithelial cell types, and, interestingly, are enveloped by a sheath of mesenchyme, comprised of myofibroblasts and smooth muscle cells, thus recapitulating an additional aspect of the ISC niche.

Aside from promising to revolutionize basic and clinical research, by serving as models of development and disease, platforms for drug discovery and toxicity screens and sources of tissue for cell-based therapies $[23,65]$, intestinal organoids complement in vivo studies in our quest to dissect the ISC 
niche and define the mechanisms whereby it exerts its influence on stem cells. Indeed, to demonstrate that Paneth cells constitute the ISC niche, as discussed above, mouse models were used in conjunction with intestinal organoids [34, 66]. Likewise, organoids have been instrumental in elucidating the roles of various genes, including R-spondin and Lgr4/5 $[67,68]$ and YAP $[69,70]$ in the regulation of ISC fate.

The format of common organoid culture models allows for relatively easy and routine manipulations of the soluble microenvironment of ISCs. Beyond a set of soluble cues, the stem cell niche also comprises adhesion and mechanical signals from the surrounding ECM [71], which are likely to be as important as morphogens and growth factors in regulating ISC fate [72-75]. Typical intestinal organoid models, however, employ Matrigel-an ECM protein-rich hydrogel derived from the Engelbreth-Holm-Swarm sarcoma-as the 3D matrix. Matrigel, while clearly providing essential adhesive and mechanical cues, without which organoid formation would not be possible, remains a black box in regard to its contribution to the ISC niche. That is, Matrigel is a complex multicomponent mixture with ill-fined and variable biochemical and biophysical properties $[76,77]$ and the specific components and mechanisms whereby this material influences ISC fate are unclear. In the following sections, we will discuss recent advances in using biomaterials and bioengineering approaches to overcome the limitations of Matrigel, secure a more holistic understanding, and introduce additional levels of control over the ISC niche.

3.2. Toward a Synthetic ISC Niche: Using Synthetic Matrices to Deconstruct the Native Intestinal ECM. Synthetic hydrogels, comprising a water-swelled polymer network, can be rendered biocompatible and biofunctional through the incorporation of essential biological signals and used as well-defined alternatives to animal-derived ECM gels, such as collagen and Matrigel [78-80]. Moreover, these materials provide a biologically "blank" 3D environment into which biochemical and biophysical factors found in native tissues can be introduced and varied in a systematic and controlled manner, thus interrogating their cellular effects and evaluating them as potential stem cell niche components.

We recently took advantage of poly(ethylene glycol) (PEG) hydrogels to identify ECM components that control ISC fate and used this knowledge to construct well-defined and tunable matrices for the culture of ISCs and intestinal organoids [81] (Figure 2). Inspired by their localization to the basement membrane of mouse and human intestinal crypts in vivo [60, 74, 82-85], we assessed the effect of laminin-111, collagen-IV, fibronectin, hyaluronic acid, and perlecan on ISC self-renewal, differentiation, and organoid formation in the context of a 3D PEG hydrogel. We found that all components enhanced ISC survival and colony formation; laminin-111, collagen-IV, and fibronectin displayed the strongest positive effects. Notably, the fibronectinderived RGD (Arg-Gly-Asp) peptide was sufficient in supporting ISC expansion in synthetic matrices. On the other hand, laminin-111 was of crucial importance for the concerted cycles of ISC self-renewal, differentiation, and morphogenesis that drive organoid formation; none of the other ECM components tested were even minimally effective. Although informative, our study examined the effects of only a handful of ECM components found in vivo. Future studies not only could take a system-level approach and expanded the number of ECM factors tested but also investigate potential interactive effects of multiple components. Sophisticated high-throughput approaches to generate and analyze multifactorial environments, which have already been used to study other stem cell systems [86, 87], seem ideally suited for further deconstructing the complexity of the ISC niche.

In addition to soluble and tethered molecular factors, ISCs in vivo experience physical signals from the microenvironment, including the mechanical properties of their surrounding ECM. The mechanical environment is now recognized as a major extrinsic regulator of multiple stem cell systems [88]. Our understanding of potential physical regulators of ISC fate is minimal, owing to the difficulty of performing controlled mechanical perturbations in both mouse models and Matrigel-based organoid culture. Nevertheless, recent in vivo studies provide clues that mechanical forces may directly control ISC proliferation in the colon [89]. We used the mechanically tunable PEG matrices to examine the effect of matrix mechanical properties on ISC expansion and organoid formation [81] and observed profound effects. In particular, we found that relatively stiff (shear modulus of $\sim 1 \mathrm{kPa}$ ) matrices were optimal for ISC expansion, whereas ISC survival and colony formation in soft matrices was exceedingly low. In contrast, ISC differentiation and organoid formation were impaired by stiff matrices and only occurred in soft ones. We again took advantage of the versatility of the PEG hydrogel system to shed light on the molecular mechanisms underlying the mechanical effects on ISC expansion and organoid formation, which, surprisingly also accounted for the seemingly contradictory influence of stiffness on these two processes. In particular, we found that the matrix mechanics regulates ISC behavior by controlling the activity of Yes-associated protein 1 (YAP), which is a known mechanotransducer in other cellular systems [90, 91], and is also required for ISC expansion and organoid formation $[69,70,81]$. We showed that stiff matrices enhance ISC colony formation by inducing nuclear translocation of YAP in single-embedded ISCs. However, continued ISC proliferation within a stiff environment led to cell confinement and compression, which in turn resulted in gradual YAP inactivation. Creating matrices that are initially stiff enough to induce YAP activation but soften in a controlled manner to prevent cell compression rescued YAP inactivation and supported both ISC expansion and organoid formation. Thus, we used modular PEG-based matrices to unveil matrix stiffness as another important component of the ISC niche [81].

3.3. Future Perspective: Using Microengineering to Establish a Homeostatic ISC Niche In Vitro. A key difference between the intestinal niche in vivo and current organoid models is not only the types of niche components available but also the mode in which they are presented. Current organoid cultures contain essential niche signals, including Wnt and Notch 

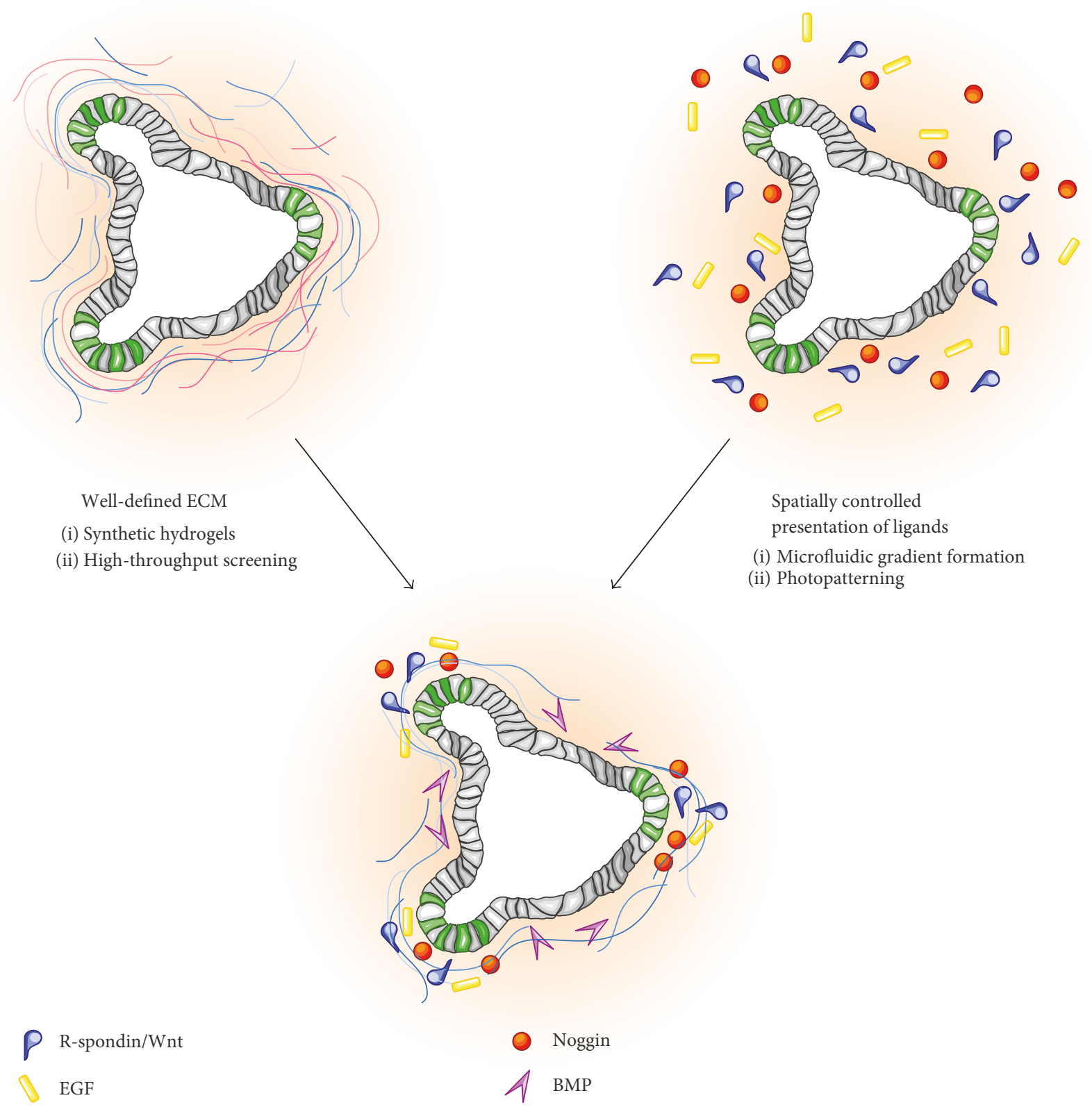

FIGURE 2: Engineering the ISC niche in vitro. Bioengineering approaches could further increase the tractability of organoid models and their fidelity to the real intestine. Synthetic matrices simplify the complexity of Matrigel and offer a powerful new toolkit with which to examine the effects of individual or combinations of ECM and mechanical niche signals. Microengineering approaches can be used to introduce spatial and temporal control over the biochemical and biophysical environment of ISCs, thereby mimicking the native niche more closely.

pathway agonists, and BMP inhibitors. However, these soluble components are included in the cell culture medium, wherein they eventually reach a uniform concentration. In contrast, master regulators of intestinal biology in vivo are presented in distinct spatiotemporal patterns, which are crucial for the regionalization of the intestine and the establishment of the ISC niche. For example, Wnt signals in vivo are produced by the subepithelial mesenchyme and Paneth cell and thus restricted to the bottom of the crypts where they are crucial for maintaining ISCs in a self-renewing state [92]. Bone morphogenetic protein (BMP) and Sonic Hedgehog (Shh) signals, are, on the other hand, enriched in the villus region, where they suppress proliferation and ensure differentiation into functional enterocytes [92]. This difference in the presentation of soluble cues may account for the fact that intestinal organoids are continuously expanding structures, with new crypt-like buds forming perpetually. Thus, intestinal organoids currently mimic a developmental or regenerative, rather than a homeostatic state, wherein ISC self-renewal is balanced by differentiation and apoptosis to establish a stable niche (the intestinal crypt).

Bioengineers have developed a number of strategies for controlling the spatiotemporal patterns of soluble and tethered cues in soft 3D media, similar to the matrices required 
for organoid formation. Microfluidically generated morphogen gradients are perhaps the most widespread approach. Here, 3D hydrogels are microstructured using lithographic [93-95] or ablative [96] techniques. The resulting channels are loaded with a molecular of interest, which forms a gradient through the surrounding permeable gel. The shape of the gradient and, thus, the spatiotemporal mode of biomolecule delivery to encapsulated cells can be controlled by varying the concentration of the molecule at the source (the channel), the flow rate, and the diffusive properties of the permeable medium. Photopatterning approaches provide even finer spatial and temporal control over the distribution of mechanical and tethered molecular cues in 3D gels. These strategies use controlled illumination to locally change the properties of hydrogels that have been engineered to contain photosensitive building blocks [97-99]. The molecular changes induced can then be used to add or remove molecules or alter the mechanical stiffness of a desired region, at a desired time. We believe that these and other approaches for controlling the spatial and temporal presentation of diffusible or immobilized cues may be useful for the generation of the separate molecular zones seen in the native intestine, which could in turn contribute toward creating a more realistic in vitro model of a stable, homeostatic ISC niche (Figure 2).

\section{Conflicts of Interest}

The authors declare that they have no conflicts of interest.

\section{References}

[1] D. H. Scoville, T. Sato, X. C. He, and L. Li, "Current view: intestinal stem cells and signaling," Gastroenterology, vol. 134, no. 3, pp. 849-864, 2008.

[2] N. Barker, J. H. van Es, J. Kuipers et al., "Identification of stem cells in small intestine and colon by marker gene Lgr5," Nature, vol. 449, no. 7165, pp. 1003-1007, 2007.

[3] F. M. Barriga, E. Montagni, M. Mana et al., "Mex3a marks a slowly dividing subpopulation of $\mathrm{Lgr}^{+}$intestinal stem cells," Cell Stem Cell, vol. 20, no. 6, pp. 801-816, 2017.

[4] R. K. Montgomery, D. L. Carlone, C. A. Richmond et al., "Mouse telomerase reverse transcriptase (mTert) expression marks slowly cycling intestinal stem cells," Proceedings of the National Academy of Sciences of the United States of America, vol. 108, no. 1, pp. 179-184, 2011.

[5] E. Sangiorgi and M. R. Capecchi, "Bmil is expressed in vivo in intestinal stem cells," Nature Genetics, vol. 40, no. 7, pp. 915-920, 2008.

[6] C. S. Potten, G. Owen, and D. Booth, "Intestinal stem cells protect their genome by selective segregation of template DNA strands," Journal of Cell Science, vol. 115, Part 11, pp. 2381-2388, 2002.

[7] N. Takeda, R. Jain, M. R. LeBoeuf, Q. Wang, M. M. Lu, and J. A. Epstein, "Interconversion between intestinal stem cell populations in distinct niches," Science, vol. 334, no. 6061, pp. 1420-1424, 2011.

[8] K. S. Yan, L. A. Chia, X. Li et al., "The intestinal stem cell markers Bmi1 and Lgr5 identify two functionally distinct populations," Proceedings of the National Academy of Sciences of the United States of America, vol. 109, no. 2, pp. 466-471, 2012.
[9] J. H. van Es, T. Sato, M. van de Wetering et al., "Dll1+ secretory progenitor cells revert to stem cells upon crypt damage," Nature Cell Biology, vol. 14, no. 10, pp. 1099-1104, 2012.

[10] O. Basak, J. Beumer, K. Wiebrands, H. Seno, A. van Oudenaarden, and H. Clevers, "Induced quiescence of Lgr5 $5^{+}$ stem cells in intestinal organoids enables differentiation of hormone-producing Enteroendocrine cells," Cell Stem Cell, vol. 20, no. 2, article e4, pp. 177-190, 2017.

[11] P. W. Tetteh, O. Basak, H. F. Farin et al., "Replacement of lost Lgr5-positive stem cells through plasticity of their enterocyte-lineage daughters," Cell Stem Cell, vol. 18, no. 2, pp. 203-213, 2016.

[12] H. Tian, B. Biehs, S. Warming et al., "A reserve stem cell population in small intestine renders Lgr5-positive cells dispensable," Nature, vol. 478, no. 7368, pp. 255-259, 2011.

[13] C. Metcalfe, N. M. Kljavin, R. Ybarra, and F. J. de Sauvage, "Lgr5 ${ }^{+}$stem cells are indispensable for radiation-induced intestinal regeneration," Cell Stem Cell, vol. 14, no. 2, pp. 149-159, 2014.

[14] J. P. Medema and L. Vermeulen, "Microenvironmental regulation of stem cells in intestinal homeostasis and cancer," Nature, vol. 474, no. 7351, pp. 318-326, 2011.

[15] C. Bonnans, J. Chou, and Z. Werb, "Remodelling the extracellular matrix in development and disease," Nature Reviews Molecular Cell Biology, vol. 15, no. 12, pp. 786-801, 2014.

[16] F. M. Watt and W. T. S. Huck, "Role of the extracellular matrix in regulating stem cell fate," Nature Reviews Molecular Cell Biology, vol. 14, no. 8, pp. 467-473, 2013.

[17] P. Ordóñez-Morán and J. Huelsken, "Lrig1: a new master regulator of epithelial stem cells," The EMBO Journal, vol. 31, no. 9, pp. 2064-2066, 2012.

[18] R. G. J. Vries, M. Huch, and H. Clevers, "Stem cells and cancer of the stomach and intestine," Molecular Oncology, vol. 4, no. 5, pp. 373-384, 2010.

[19] I. V. Pinchuk, R. C. Mifflin, J. I. Saada, and D. W. Powell, "Intestinal mesenchymal cells," Current Gastroenterology Reports, vol. 12, no. 5, pp. 310-318, 2010.

[20] L. G. van der Flier, M. E. van Gijn, P. Hatzis et al., "Transcription factor achaete scute-like 2 controls intestinal stem cell fate," Cell, vol. 136, no. 5, pp. 903-912, 2009.

[21] T. Sato, R. G. Vries, H. J. Snippert et al., "Single Lgr5 stem cells build crypt-villus structures in vitro without a mesenchymal niche," Nature, vol. 459, no. 7244, pp. 262-265, 2009.

[22] A. Ootani, E. Sangiorgi, Q. T. Ho et al., "Sustained in vitro intestinal epithelial culture within a Wnt-dependent stem cell niche," Nature Medicine, vol. 15, no. 6, pp. 701-706, 2009.

[23] T. Sato and H. Clevers, "Growing self-organizing mini-guts from a single intestinal stem cell: mechanism and applications," Science, vol. 340, no. 6137, pp. 1190-1194, 2013.

[24] T. Sato, D. E. Stange, M. Ferrante et al., "Long-term expansion of epithelial organoids from human colon, adenoma, adenocarcinoma, and Barrett's epithelium," Gastroenterology, vol. 141, no. 5, pp. 1762-1772, 2011.

[25] P. Jung, T. Sato, A. Merlos-Suárez et al., "Isolation and in vitro expansion of human colonic stem cells," Nature Medicine, vol. 17, no. 10, pp. 1225-1227, 2011.

[26] T. Fevr, S. Robine, D. Louvard, and J. Huelsken, "Wnt/ beta-catenin is essential for intestinal homeostasis and maintenance of intestinal stem cells," Molecular and Cellular Biology, vol. 27, no. 21, pp. 7551-7559, 2007. 
[27] V. Korinek, N. Barker, P. Moerer et al., "Depletion of epithelial stem-cell compartments in the small intestine of mice lacking Tcf-4," Nature Genetics, vol. 19, no. 4, pp. 379-383, 1998.

[28] D. Pinto, A. Gregorieff, H. Begthel, and H. Clevers, "Canonical Wnt signals are essential for homeostasis of the intestinal epithelium," Genes \& Development, vol. 17, no. 14, pp. 17091713, 2003.

[29] K.-A. Kim, M. Kakitani, J. Zhao et al., "Mitogenic influence of human R-spondin1 on the intestinal epithelium," Science, vol. 309, no. 5738, pp. 1256-1259, 2005.

[30] L. K. Su and K. W. Kinzler, "Multiple intestinal neoplasia caused by a mutation in the murine homolog of the APC gene," Science, vol. 256, no. 5057, pp. 668-670, 1992.

[31] J. Hilkens, N. C. Timmer, M. Boer et al., "RSPO3 expands intestinal stem cell and niche compartments and drives tumorigenesis," Gut, vol. 66, no. 6, pp. 1095-1105, 2017.

[32] E. Batlle, J. T. Henderson, H. Beghtel et al., "Beta-catenin and TCF mediate cell positioning in the intestinal epithelium by controlling the expression of EphB/ephrinB," Cell, vol. 111, no. 2, pp. 251-263, 2002.

[33] J. Holmberg, M. Genander, M. M. Halford et al., "EphB receptors coordinate migration and proliferation in the intestinal stem cell niche," Cell, vol. 125, no. 6, pp. 11511163, 2006.

[34] T. Sato, J. H. van Es, H. J. Snippert et al., "Paneth cells constitute the niche for Lgr5 stem cells in intestinal crypts," Nature, vol. 469, no. 7330, pp. 415-418, 2011.

[35] S. S. Poulsen, E. Nexø, P. S. Olsen, J. Hess, and P. Kirkegaard, "Immunohistochemical localization of epidermal growth factor in rat and man," Histochemistry, vol. 85, no. 5, pp. 389-394, 1986.

[36] T.-H. Kim, S. Escudero, and R. A. Shivdasani, "Intact function of Lgr5 receptor-expressing intestinal stem cells in the absence of Paneth cells," Proceedings of the National Academy of Sciences of the United States of America, vol. 109, no. 10, pp. 3932-3937, 2012.

[37] M. E. Rothenberg, Y. Nusse, T. Kalisky et al., "Identification of a cKit(+) colonic crypt base secretory cell that supports Lgr5(+) stem cells in mice," Gastroenterology, vol. 142, no. 5, article e6, pp. 1195-1205, 2012.

[38] N. Sasaki, N. Sachs, K. Wiebrands et al., "Reg4+ deep crypt secretory cells function as epithelial niche for Lgr $5^{+}$stem cells in colon," Proceedings of the National Academy of Sciences of the United States of America, vol. 113, no. 37, pp. E5399E5407, 2016.

[39] M. Schewe, P. F. Franken, A. Sacchetti et al., "Secreted phospholipases A2 are intestinal stem cell niche factors with distinct roles in homeostasis, inflammation, and cancer," Cell Stem Cell, vol. 19, no. 1, pp. 38-51, 2016.

[40] S. Fre, M. Huyghe, P. Mourikis, S. Robine, D. Louvard, and S. Artavanis-Tsakonas, "Notch signals control the fate of immature progenitor cells in the intestine," Nature, vol. 435, no. 7044, pp. 964-968, 2005.

[41] L. Pellegrinet, V. Rodilla, Z. Liu et al., "Dll1- and dll4-mediated notch signaling are required for homeostasis of intestinal stem cells," Gastroenterology, vol. 140, no. 4, pp. 1230-1240, 2011, e1-7.

[42] K. L. VanDussen, A. J. Carulli, T. M. Keeley et al., "Notch signaling modulates proliferation and differentiation of intestinal crypt base columnar stem cells," Development (Cambridge, England), vol. 139, no. 3, pp. 488-497, 2012.
[43] H. Tian, B. Biehs, C. Chiu et al., "Opposing activities of notch and Wnt signaling regulate intestinal stem cells and gut homeostasis," Cell Reports, vol. 11, no. 1, pp. 33-42, 2015.

[44] O. Riccio, M. E. van Gijn, A. C. Bezdek et al., "Loss of intestinal crypt progenitor cells owing to inactivation of both Notch1 and Notch 2 is accompanied by derepression of CDK inhibitors p27Kip1 and p57Kip2," EMBO Reports, vol. 9, no. 4, pp. 377383,2008

[45] J. H. van Es, N. de Geest, M. van de Born, H. Clevers, and B. A. Hassan, "Intestinal stem cells lacking the Math1 tumour suppressor are refractory to Notch inhibitors," Nature Communications, vol. 1, p. 18, 2010.

[46] J. H. van Es, M. E. van Gijn, O. Riccio et al., "Notch/gammasecretase inhibition turns proliferative cells in intestinal crypts and adenomas into goblet cells," Nature, vol. 435, no. 7044, pp. 959-963, 2005.

[47] V. W. Y. Wong, D. E. Stange, M. E. Page et al., "Lrig1 controls intestinal stem-cell homeostasis by negative regulation of ErbB signalling," Nature Cell Biology, vol. 14, no. 4, pp. 401-408, 2012.

[48] N. Normanno, A. De Luca, C. Bianco et al., "Epidermal growth factor receptor (EGFR) signaling in cancer," Gene, vol. 366, no. 1, pp. 2-16, 2006.

[49] C. Kosinski, V. S. Li, A. S. Chan et al., "Gene expression patterns of human colon tops and basal crypts and BMP antagonists as intestinal stem cell niche factors," Proceedings of the National Academy of Sciences of the United States of America, vol. 104, no. 39, pp. 15418-15423, 2007.

[50] X. C. He, J. Zhang, W. G. Tong et al., "BMP signaling inhibits intestinal stem cell self-renewal through suppression of Wnt-beta-catenin signaling," Nature Genetics, vol. 36, no. 10, pp. 1117-1121, 2004.

[51] A.-P. G. Haramis, H. Begthel, M. van den Born et al., "De novo crypt formation and juvenile polyposis on BMP inhibition in mouse intestine," Science, vol. 303, no. 5664, pp. 1684-1686, 2004.

[52] W. J. Zacharias, X. Li, B. B. Madison et al., "Hedgehog is an anti-inflammatory epithelial signal for the intestinal lamina propria," Gastroenterology, vol. 138, no. 7, pp. 2368-2377, 2010, 2377.e1-4.

[53] H. F. Farin, J. H. Van Es, and H. Clevers, "Redundant sources of Wnt regulate intestinal stem cells and promote formation of Paneth cells," Gastroenterology, vol. 143, no. 6, article e7, pp. 1518-1529, 2012.

[54] A. Gregorieff and H. Clevers, "In situ hybridization to identify gut stem cells," Current Protocols in Stem Cell Biology, vol. 34, pp. 2F.1.1-2F.111, 2015.

[55] E. Kang, M. Yousefi, and S. Gruenheid, "R-spondins are expressed by the intestinal stroma and are differentially regulated during Citrobacter rodentium- and DSSinduced colitis in mice," PloS One, vol. 11, no. 4, article e0152859, 2016.

[56] Z. Kabiri, G. Greicius, B. Madan et al., "Stroma provides an intestinal stem cell niche in the absence of epithelial Wnts," Development (Cambridge, England), vol. 141, no. 11, pp. 2206-2215, 2014.

[57] R. Aoki, M. Shoshkes-Carmel, N. Gao et al., "Foxl1-expressing mesenchymal cells constitute the intestinal stem cell niche," Cellular and Molecular Gastroenterology and Hepatology, vol. 2, no. 2, pp. 175-188, 2016. 
[58] H. Horiguchi, M. Endo, K. Kawane et al., "ANGPTL2 expression in the intestinal stem cell niche controls epithelial regeneration and homeostasis," The EMBO Journal, vol. 36, no. 4, pp. 409-424, 2017.

[59] B. S. Sailaja, X. C. He, and L. Li, "The regulatory niche of intestinal stem cells," The Journal of Physiology, vol. 594, no. 17, pp. 4827-4836, 2016.

[60] P. Simon-Assmann, B. Duclos, V. Orian-Rousseau et al., "Differential expression of laminin isoforms and alpha 6beta 4 integrin subunits in the developing human and mouse intestine," Developmental dynamics: an official publication of the American Association of Anatomists, vol. 201, no. 1, pp. 71-85, 1994.

[61] Y. Sasai, "Next-generation regenerative medicine: organogenesis from stem cells in 3D culture," Cell Stem Cell, vol. 12, no. 5, pp. 520-530, 2013.

[62] Y. Sasai, "Cytosystems dynamics in self-organization of tissue architecture," Nature, vol. 493, no. 7432, pp. 318-326, 2013.

[63] D. Grün, A. Lyubimova, L. Kester et al., "Single-cell messenger RNA sequencing reveals rare intestinal cell types," Nature, vol. 525, no. 7568, pp. 251-255, 2015.

[64] J. R. Spence, C. N. Mayhew, S. A. Rankin et al., "Directed differentiation of human pluripotent stem cells into intestinal tissue in vitro," Nature, vol. 470, no. 7332, pp. 105-109, 2011.

[65] M. A. Lancaster and J. A. Knoblich, "Organogenesis in a dish: modeling development and disease using organoid technologies," Science, vol. 345, no. 6194, article 1247125, 2014.

[66] Ö. H. Yilmaz, P. Katajisto, D. W. Lamming et al., "mTORC1 in the Paneth cell niche couples intestinal stem-cell function to calorie intake," Nature, vol. 486, no. 7404, pp. 490-495, 2012.

[67] W. de Lau, N. Barker, T. Y. Low et al., "Lgr5 homologues associate with Wnt receptors and mediate R-spondin signalling," Nature, vol. 476, no. 7360, pp. 293-297, 2011.

[68] R. C. Mustata, T. Van Loy, A. Lefort et al., "Lgr4 is required for Paneth cell differentiation and maintenance of intestinal stem cells ex vivo," EMBO Reports, vol. 12, no. 6, pp. 558564, 2011.

[69] L. Azzolin, T. Panciera, S. Soligo et al., "YAP/TAZ incorporation in the $\beta$-catenin destruction complex orchestrates the Wnt response," Cell, vol. 158, no. 1, pp. 157-170, 2014.

[70] A. Gregorieff, Y. Liu, M. R. Inanlou, Y. Khomchuk, and J. L. Wrana, "Yap-dependent reprogramming of Lgr5(+) stem cells drives intestinal regeneration and cancer," Nature, vol. 526, no. 7575, pp. 715-718, 2015.

[71] M. P. Lutolf, P. M. Gilbert, and H. M. Blau, "Designing materials to direct stem-cell fate," Nature, vol. 462, no. 7272, pp. 433-441, 2009.

[72] R. G. Jones, X. Li, P. D. Gray et al., "Conditional deletion of betal integrins in the intestinal epithelium causes a loss of Hedgehog expression, intestinal hyperplasia, and early postnatal lethality," The Journal of Cell Biology, vol. 175, no. 3, pp. 505-514, 2006.

[73] Z. X. Mahoney, T. S. Stappenbeck, and J. H. Miner, "Laminin alpha 5 influences the architecture of the mouse small intestine mucosa," Journal of Cell Science, vol. 121, Part 15, pp. 24932502, 2008.

[74] S. Yamamoto, H. Nakase, M. Matsuura et al., "Heparan sulfate on intestinal epithelial cells plays a critical role in intestinal crypt homeostasis via Wnt/ $\beta$-catenin signaling," American Journal of Physiology Gastrointestinal and Liver Physiology, vol. 305, no. 3, pp. G241-G249, 2013.
[75] T. E. Riehl, S. Santhanam, L. Foster, M. Ciorba, and W. F. Stenson, "CD44 and TLR4 mediate hyaluronic acid regulation of Lgr $5^{+}$stem cell proliferation, crypt fission, and intestinal growth in postnatal and adult mice," American Journal of Physiology Gastrointestinal and Liver Physiology, vol. 309, no. 11, pp. G874-G887, 2015.

[76] H. K. Kleinman and G. R. Martin, "Matrigel: basement membrane matrix with biological activity," Seminars in Cancer Biology, vol. 15, no. 5, pp. 378-386, 2005.

[77] S. Vukicevic, H. K. Kleinman, F. P. Luyten, A. B. Roberts, N. S. Roche, and A. H. Reddi, "Identification of multiple active growth factors in basement membrane Matrigel suggests caution in interpretation of cellular activity related to extracellular matrix components," Experimental Cell Research, vol. 202, no. 1, pp. 1-8, 1992.

[78] D. Seliktar, "Designing cell-compatible hydrogels for biomedical applications,” Science, vol. 336, no. 6085, pp. 1124-1128, 2012.

[79] M. P. Lutolf and J. A. Hubbell, "Synthetic biomaterials as instructive extracellular microenvironments for morphogenesis in tissue engineering," Nature Biotechnology, vol. 23, no. 1, pp. 47-55, 2005.

[80] M. W. Tibbitt and K. S. Anseth, "Hydrogels as extracellular matrix mimics for 3D cell culture," Biotechnology and Bioengineering, vol. 103, no. 4, pp. 655-663, 2009.

[81] N. Gjorevski, N. Sachs, A. Manfrin et al., "Designer matrices for intestinal stem cell and organoid culture," Nature, vol. 539, no. 7630, pp. 560-564, 2016, 24.

[82] A. D. Arcangelis, P. Neuville, R. Boukamel, O. Lefebvre, M. Kedinger, and P. Simon-Assmann, "Inhibition of laminin alpha 1-chain expression leads to alteration of basement membrane assembly and cell differentiation," The Journal of Cell Biology, vol. 133, no. 2, pp. 417-430, 1996.

[83] P. Simo, P. Simon-Assmann, F. Bouziges et al., "Changes in the expression of laminin during intestinal development," Development Cambridge, England, vol. 112, no. 2, pp. 477-487, 1991.

[84] P. Simon-Assmann, M. Kedinger, A. D. Arcangelis, V. Rousseau, and P. Simo, "Extracellular matrix components in intestinal development," Experientia, vol. 51, no. 9-10, pp. 883-900, 1995.

[85] F. Wang, D. Scoville, X. C. He et al., "Isolation and characterization of intestinal stem cells based on surface marker combinations and colony-formation assay," Gastroenterology, vol. 145 , no. 2, pp. 383-395, 2013, e1-21.

[86] S. Gobaa, S. Hoehnel, M. Roccio, A. Negro, S. Kobel, and M. P. Lutolf, "Artificial niche microarrays for probing single stem cell fate in high throughput," Nature Methods, vol. 8, no. 11, pp. 949-955, 2011.

[87] A. Ranga, S. Gobaa, Y. Okawa, K. Mosiewicz, A. Negro, and M. P. Lutolf, "3D niche microarrays for systems-level analyses of cell fate,” Nature Communications, vol. 5, p. 4324, 2014.

[88] F. Guilak, D. M. Cohen, B. T. Estes, J. M. Gimble, W. Liedtke, and C. S. Chen, "Control of stem cell fate by physical interactions with the extracellular matrix," Cell Stem Cell, vol. 5, no. 1, pp. 17-26, 2009.

[89] M. E. Fernández-Sánchez, S. Barbier, J. Whitehead et al., "Mechanical induction of the tumorigenic $\beta$-catenin pathway by tumour growth pressure," Nature, vol. 523, no. 7558, pp. 92-95, 2015.

[90] M. Aragona, T. Panciera, A. Manfrin et al., "A mechanical checkpoint controls multicellular growth through YAP/TAZ 
regulation by actin-processing factors," Cell, vol. 154, no. 5, pp. 1047-1059, 2013.

[91] S. Dupont, L. Morsut, M. Aragona et al., "Role of YAP/TAZ in mechanotransduction," Nature, vol. 474, no. 7350, pp. 179-183, 2011.

[92] C. Crosnier, D. Stamataki, and J. Lewis, "Organizing cell renewal in the intestine: stem cells, signals and combinatorial control," Nature Reviews Genetics, vol. 7, no. 5, pp. 349-359, 2006.

[93] N. W. Choi, M. Cabodi, B. Held, J. P. Gleghorn, L. J. Bonassar, and A. D. Stroock, "Microfluidic scaffolds for tissue engineering," Nature Materials, vol. 6, no. 11, pp. 908-915, 2007.

[94] Y. Choi, M. A. McClain, M. C. LaPlaca, A. B. Frazier, and M. G. Allen, "Three dimensional MEMS microfluidic perfusion system for thick brain slice cultures," Biomedical Microdevices, vol. 9, no. 1, pp. 7-13, 2007.

[95] Y. Zheng, J. Chen, M. Craven et al., "In vitro microvessels for the study of angiogenesis and thrombosis," Proceedings of the National Academy of Sciences of the United States of America, vol. 109, no. 24, pp. 9342-9347, 2012.

[96] N. Brandenberg and M. P. Lutolf, "In situ patterning of microfluidic networks in 3D cell-laden hydrogels," Advanced materials Deerfield Beach Fla, vol. 28, no. 34, pp. 7450-7456, 2016.

[97] C. A. DeForest and K. S. Anseth, "Photoreversible patterning of biomolecules within click-based hydrogels," Angewandte Chemie (International Ed. In English), vol. 51, no. 8, pp. 1816-1819, 2012.

[98] K. A. Mosiewicz, L. Kolb, A. J. van der Vlies et al., "In situ cell manipulation through enzymatic hydrogel photopatterning," Nature Materials, vol. 12, no. 11, pp. 1072-1078, 2013.

[99] R. G. Wylie, S. Ahsan, Y. Aizawa, K. L. Maxwell, C. M. Morshead, and M. S. Shoichet, "Spatially controlled simultaneous patterning of multiple growth factors in threedimensional hydrogels," Nature Materials, vol. 10, no. 10, pp. 799-806, 2011. 

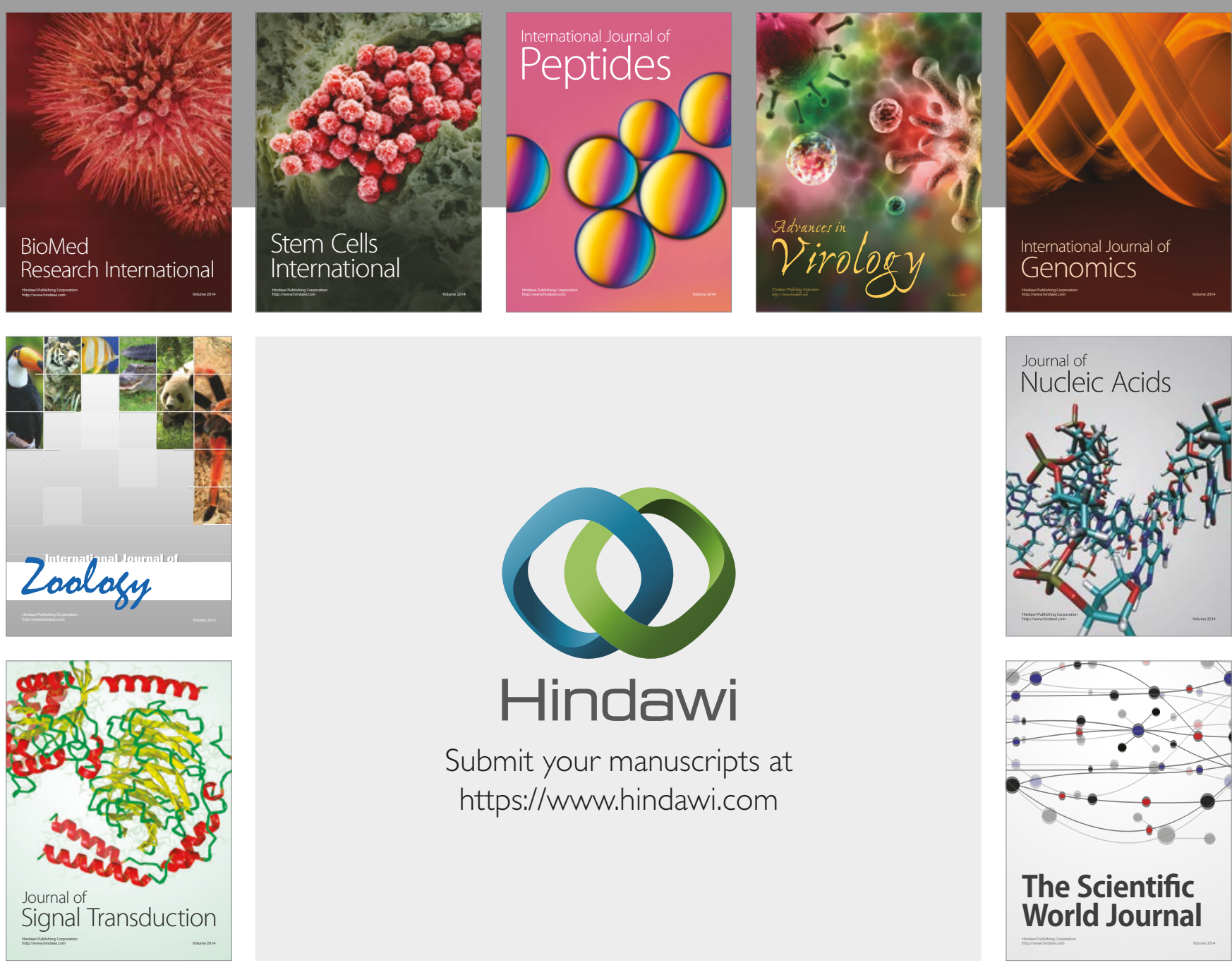

Submit your manuscripts at

https://www.hindawi.com
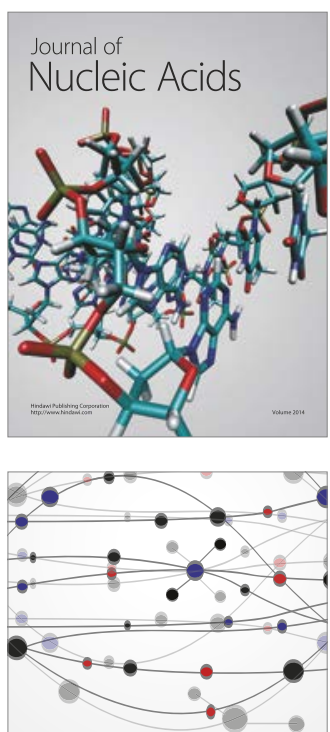

The Scientific World Journal

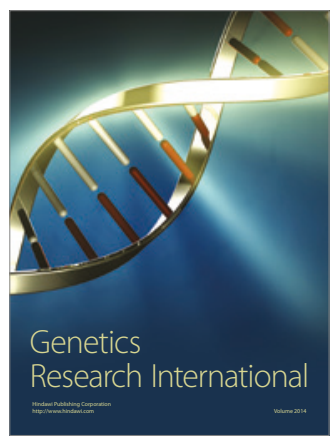

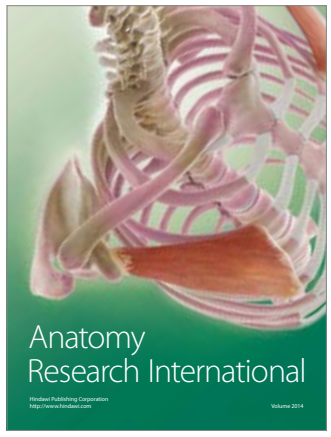

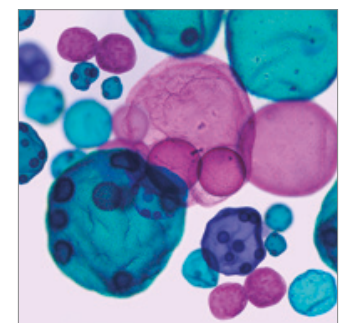

International Journal of Microbiology
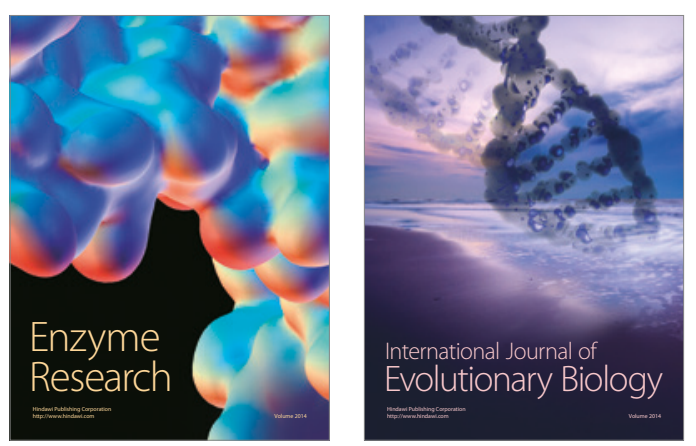
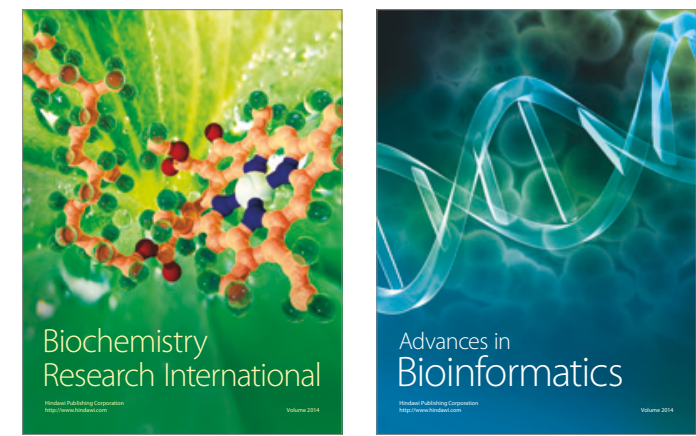

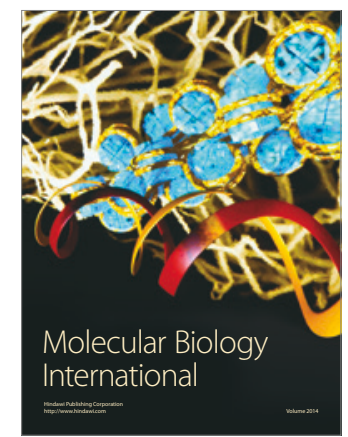

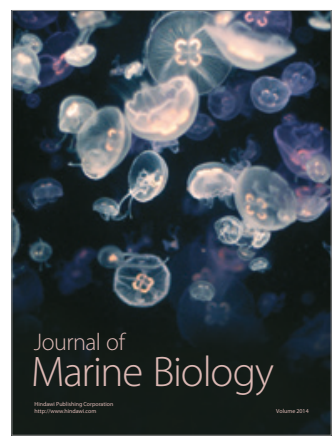

